\title{
Improving Registration Using Multi-Channel Diffeomorphic Demons Combined with Certainty Maps
}

\author{
Daniel Forsberg ${ }^{1,2,3}$, Yogesh Rathi ${ }^{4}$, Sylvain Bouix ${ }^{4}$, Demian Wassermann ${ }^{4}$, \\ Hans Knutsson ${ }^{2,3}$, and Carl-Fredrik Westin ${ }^{5}$ \\ 1 Sectra Imtec, Linköping, Sweden \\ 2 Department of Biomedical Engineering, Linköping University, Sweden \\ 3 Center for Medical Image Science and Visualization (CMIV), \\ Linköping University, Sweden \\ 4 Psychiatry Neuroimaging Laboratory, Brigham and Womens Hospital, \\ Harvard Medical School, Boston, MA, USA \\ 5 Laboratory of Mathematics in Imaging, Brigham and Womens Hospital, \\ Harvard Medical School, Boston, MA, USA
}

\begin{abstract}
The number of available imaging modalities increases both in clinical practice and in clinical studies. Even though data from multiple modalities might be available, image registration is typically only performed using data from a single modality. In this paper, we propose using certainty maps together with multi-channel diffeomorphic demons in order to improve both accuracy and robustness when performing image registration. The proposed method is evaluated using DTI data, multiple region overlap measures and a fiber bundle similarity metric.
\end{abstract}

\section{Introduction}

Image registration is a well-known concept within the medical image domain and has proven to be useful in a number of situations and applications. The basic idea of image registration is to find a displacement field $\boldsymbol{d}$ that aligns a source image $S$ with a target image $T$. The research related to image registration is vast and the available methods are many [4]. However, up until today, most research regarding image registration has been related to scalar image registration, although there has been a constant increase of available imaging modalities both for clinical practice and clinical studies. Often each modality has its own unique and, thus, complementary information depicting the underlying anatomy, see Fig. 1 for an example of this. Thus, combining multiple modalities and performing multivariate image registration is likely to improve both accuracy and robustness.

The primary challenge in registration of multi-variate data lies in finding a suitable way of fusing information from different modalities. The first approach for multi-variate image registration is to expand scalar similarity metrics to their corresponding multi-variate versions, e.g. multi-variate mutual information. However, the computational costs associated with estimating multi-variate 

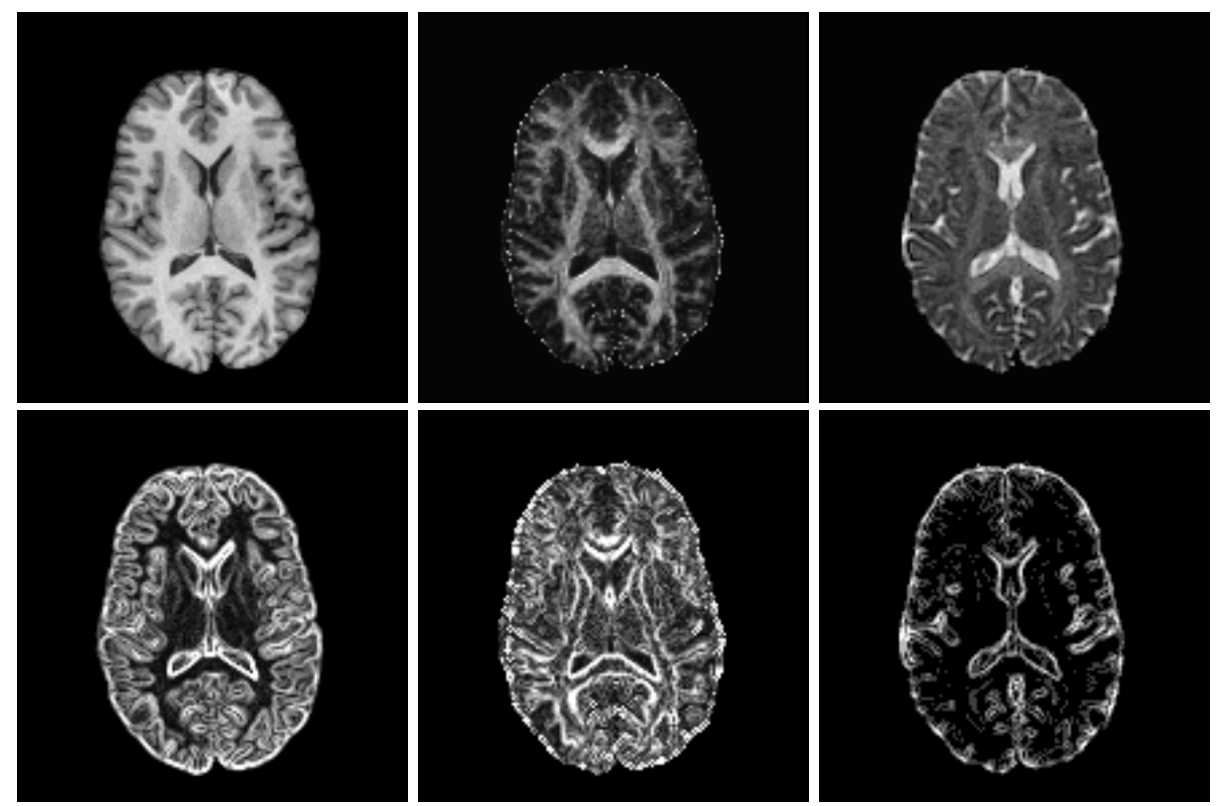

Fig. 1. Comparing a T1 image with images depicting fractional anisotropy and parallel diffusivity reveals the complimentary information provided by different modalities. This becomes more apparent when observing the gradient of the corresponding images and especially in regards of white matter and gray matter.

mutual information makes it too cumbersome to use and calls for different assumptions or approximations to be made [11,12].

A common approach is to fuse the information from the multi-variate data into a single scalar, typically done for diffusion tensor imaging (DTI) data where fractional anisotropy (FA) is used, and then to apply standard scalar-based registration methods. Li and Verma [7] introduced a novel multi-channel method, which contrary to fusing multi-variate data at image/intensity level, instead fuses information on a feature level. Another novelty in their suggestion is that they use independent component analysis and a choose-max approach to fuse and extract relevant information.

Another approach is to use multi-channel registration. Typically, an arbitrary number of channels can be used with arbitrary scalar valued images in each channel (e.g. intensity values, scalar values derived from the tensor or feature values). This approach was explored by Guimond et al. and by Parket al. [3, 8], where they used a multi-channel registration method primarily influenced by the demons algorithm. Avant et al. [1] presented the method multi-variate symmetric normalization (MVSyN), which is an extension of the SyN registration method to handle multiple channels. In common for these methods, is that each channel independently estimates an update field for each variate, which are then simply averaged to update the full displacement field. 
In this paper we expand a multi-channel version of the well-known demons algorithm to also include certainties of the channel-wise estimated update fields, in order to create a spatially adaptive combination of the update fields by use of weighted averaging. The multi-channel registration is compared with single channel registration on a set of DTI data using various metrics for region overlap and a metric for fiber bundle similarity.

\section{Multi-channel diffemorphic demons with weighted averaging}

The demons algorithm was introduced by Thirion in 1995 [13] and has since then gone through a number of evolutionary steps, where one of the more recent is the extension of demons to include diffeomorphic deformations [14]. The basic diffeomorphic demons can be explained as follows:

1. Set $\boldsymbol{d}=\mathbf{0}$ and let $D=S \circ \boldsymbol{d}$.

2. Estimate an update field $\boldsymbol{u}$ as:

$$
\boldsymbol{u}=\frac{(D-T) \nabla T}{\nabla T^{2}+(D-T)^{2}}
$$

3. If fluid regularization, update $\boldsymbol{u}$ with $\boldsymbol{u} * g_{\text {fluid }}$.

4. Add $\boldsymbol{u}$ to displacement field $\mathbf{d}$ using compositive field accumulation and the exponential update field (ensures a diffeomorphic deformation):

$$
\boldsymbol{d}=\boldsymbol{d} \circ \exp (\boldsymbol{u})
$$

5. If elastic regularization, update $\mathbf{d}$ with $\boldsymbol{d} * g_{\text {elastic }}$.

6. Update $D$.

$$
D=S \circ \boldsymbol{d}
$$

7. Repeat step 2-6 until convergence.

The demons has previously been extended to handle multi-variate data, see $[3,9]$. The suggestion by Guimond et al. is somewhat ad hoc, where the update fields are simply averaged between the different channels, whereas the suggestion by Peyrat et al. is more formally derived but where the resulting solution is rather similar.

\subsection{Incorporating local certainty}

In this work we will use the suggestion by Guimond et al. to average the update fields of the different channels, but we will extend their suggestion with the use of channel-specific certainty maps, in order to replace the standard averaging with weighted averaging. Thus, step 2, in the above description, will be replaced with the following sub-steps: 
- Estimate an update field $\boldsymbol{u}_{\boldsymbol{i}}$ for each channel coupled with a certainty $c_{i}$ :

$$
\begin{aligned}
\boldsymbol{u}_{\boldsymbol{i}} & =\frac{\left(D_{i}-T_{i}\right) \nabla T_{i}}{\nabla T_{i}^{2}+\left(D_{i}-T_{i}\right)^{2}} \\
c_{i} & =\left\|\nabla T_{i}^{T} \nabla T_{i}\right\|
\end{aligned}
$$

- Normalize the certainty $c_{i}$.

$$
\hat{c}_{i}=\frac{c_{i}}{\max c_{i}}
$$

- Employ weighted averaging to the channel-specific update fields using their respective certainty maps.

$$
\boldsymbol{u}=\frac{\sum_{i} \hat{c}_{i} \boldsymbol{u}_{\boldsymbol{i}}}{\sum_{i} \hat{c}_{i}}
$$

The reason for using weighted averaging is to allow channels with high certainty to dominate the combination of the channel-specific update fields, instead of just averaging them. The usefulness of incorporating certainty into various signal/image processing operations has been shown earlier, e.g. normalized convolution by Knutsson and Westin [5]. Some examples of utilizing image registration can be found in work by Kybic and by Risholm et al. [6,10], however, the usage of certainty in image registration remains sparse. In case of the demons, and as in the case of many other registration algorithms, the reliability of the update field is usually the highest where the structural content is high, hence, we suggest to use the norm of structure tensor as certainty measure, here estimated as the outer product of the gradient.

\section{$3 \quad$ Experiments and results}

\subsection{Evaluation metrics}

Evaluating image registration is a non-trivial task because of the lack of a gold standard. A single metric can seldom capture all aspects of a successful registration. Therefore, in this paper we make use of a number of different metrics, evaluating various aspects of the registration results.

The overall registration accuracy of the brain is evaluated using two overlap measures, quantifying how well the labeled source and target volumes overlap with each other. The first measure is target overlap $(T O)$, which is the intersection between similarly labeled regions $r$ in $S$ and $T$ divided by the volume of the regions in $T$.

$$
T O=\frac{\sum_{r}\left|T_{r} \cap S_{r}\right|}{\sum_{r}\left|T_{r}\right|}
$$

where $\|$ denotes the volume in voxels. The second measure is union overlap $U O$, which is the intersection of $S_{r}$ and $T_{r}$ over their union.

$$
U O=\frac{\sum_{r}\left|T_{r} \cap S_{r}\right|}{\sum_{r}\left|T_{r} \cup S_{r}\right|}
$$


To complement these agreement measures, false negative and false positive errors were also computed. For a given region $r$, a false negative error $F N$ is a measure of how much of the target region that has been missed. The false negative error is given as the volume of the target region outside of the source region divided by the volume of the target region.

$$
F N=\frac{\sum_{r}\left|T_{r} \backslash S_{r}\right|}{\sum_{r}\left|T_{r}\right|}
$$

A false positive error $F P$, on the other hand, measures how much of the volume outside the target region that has been incorrectly labeled as a part of the region. This is given as the volume of the source region outside of the corresponding target region divided by the volume of the source region.

$$
F P=\frac{\sum_{r}\left|S_{r} \backslash T_{r}\right|}{\sum_{r}\left|S_{r}\right|}
$$

To better evaluate the registration in terms of fiber tracts, since we are using DTI data, we utilize a similarity measure developed by Wassermann et al. [16], where each fiber tract is blurred using Gaussian Processes. Based upon the blurred fibers, it is possible to create a representation of the fiber bundles for which an inner product between two fiber bundles $F$ and $F^{\prime}$ can be defined, $\left.<F, F^{\prime}\right\rangle$. With an inner product defined, a fiber bundle similarity measure, ranging from 0 to 1 , is easily constructed as:

$$
<F, F^{\prime}>_{N}=\frac{<F, F^{\prime}>}{\|F\|\left\|F^{\prime}\right\|}
$$

where

$$
\|F\|^{2}=<F, F>
$$

\subsection{Data acquisition}

The data consisted of 10 healthy subjects, where diffusion weighted images (DWI) scans had been previously acquired on a 3-T GE system using an echo planar imaging (EPI) DWI sequence. The following scan parameters were used: TR $17000 \mathrm{~ms}$, TE $78 \mathrm{~ms}$, FOV $24 \mathrm{~cm}, 144 \times 144$ encoding steps, $1.7 \mathrm{~mm}$ slice thickness. All scans had 85 axial slices parallel to the ACPC line covering the whole brain. Along with DWI images, T1 and T2 images were also acquired.

\subsection{Data pre-prcessing}

The scalar DTI measures fractional anisotropy (FA) and parallel diffusivity (PD) where estimated from the DWI data.

The labeling of different brain regions (180 different cortical and subcortical regions) was achieved using the Freesurfer software ${ }^{6}$ A T2 image, which was in

\footnotetext{
${ }^{6}$ surfer.nmr.mgh.harvard.edu
} 
the same coordinate space as the T1 image, was then diffeomorphically registered to the baseline $(S 0)$ diffusion weighted image of the same subject using FSL software's nonlinear image registration tool (FNIRT). The obtained transform was applied to the T1 image and the label map, to obtain a T1 image and a label map in the coordinate space of the diffusion images.

The tractography was done using the Slicer software ${ }^{7}$, where corpus callosum of each subject was handsegmented and then used as seeding points for standard single-tensor tractography.

\subsection{Evaluation}

To evaluate the suggested method, one subject was selected as target and the nine remaining subjects where then individually registered to the target subject. The obtained displacement fields were applied to the labels maps and the fiber bundles. The deformed label maps and fiber bundles were then compared with the target's label map and fiber bundle, using the previously described metrics. The evaluation was done using both single channel T1 and FA, multi-channel T1/FA and T1/FA/PD registration.

The results of the evaluation are presented in Table 1 and are easy to interpret, multi-channel image registration with weighted averaging improves both accuracy and robustness, albeit the improvements cannot considered to be significant. Example slices of the results are depicted in Fig. 2.

Table 1. Summary of the results from the evaluation of multi-channel registration. The results are consistently showing better accuracy and robustness for multi-channel registration, albeit minor.

\begin{tabular}{|c|c|c|c|c|c|}
\hline Channel combination & $T O$ & $U O$ & $F N$ & $F P$ & $\left\langle F_{T}, F_{D}\right\rangle$ \\
\hline T1 & $0.50 \pm 0.06$ & $0.34 \pm 0.05$ & $0.50 \pm 0.06$ & $0.50 \pm 0.06$ & $0.52 \pm 0.12$ \\
FA & $0.47 \pm 0.04$ & $0.31 \pm 0.04$ & $0.53 \pm 0.04$ & $0.52 \pm 0.05$ & $0.55 \pm 0.09$ \\
T1 and FA & $0.52 \pm 0.05$ & $0.35 \pm 0.04$ & $0.48 \pm 0.05$ & $0.48 \pm 0.05$ & $0.55 \pm 0.12$ \\
T1, FA and PD & $0.52 \pm 0.04$ & $0.35 \pm 0.04$ & $0.48 \pm 0.05$ & $0.48 \pm 0.04$ & $0.55 \pm 0.12$ \\
\hline
\end{tabular}

\section{Discussion}

It is interesting to note that these results are similar to the ones achieved by Guimond et al. and by Park et al. $[3,8]$ where they observed an improvement when using a multi-channel demons registration but without showing it to be significant. The same can be found in [1]. In this context it also worth noting that, in a recent evaluation of various registration algorithms on DTI data [15], using both single-variate and multi-variate registration methods, the single-variate

\footnotetext{
7 www.slicer.org
} 

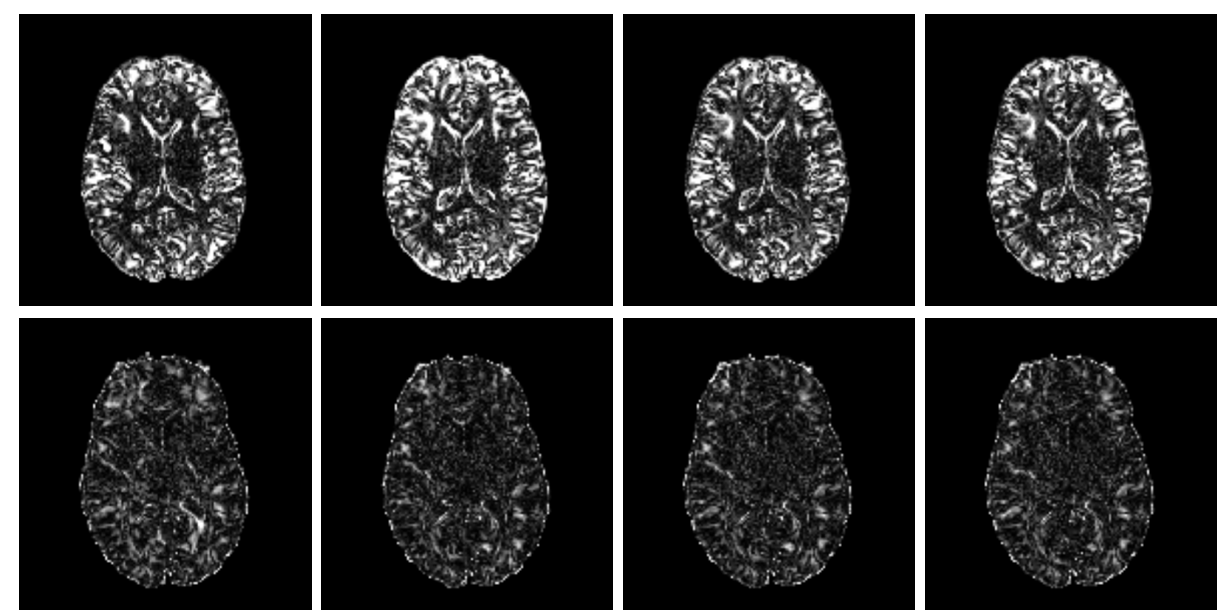

Fig. 2. Absolute difference of target and deformed T1 (top row) and FA (bottom row) slices using (from left) single channel T1 and FA, and multi-channel T1/FA and T1/FA/PD registration. Although the differences are not large, it can be observed that the multi-channel solutions performs better than the single channel solutions.

methods often performed on the same level and sometimes even better than the multi-variate methods.

In order to improve the results, various approaches were attempted, such as; changing certainty measure (magnitude of the gradient), adding a sigmoid mapping of the certainty and relaxing the regularization. In our experiments, the relaxing of the regularization showed the greatest impact. The difference between the single-channel and multi-channel increased when removing the diffeomorphic constraint but to the cost of a very irregular deformation and was therefore refrained from. A possible remedy here, would be to employ adaptive anisotropic regularization instead of isotropic Gaussian regularization [2].

A justified question at this moment, is whether multi-channel registration adds something significant to the end result or not. As the results in this paper and in $[1,3,8]$ show a consistent improvement when utilizing multi-channel registration it is reasonable to argue that multi-channel registration is valuable, however, when fusing the information more elaborate approaches must be chosen, e.g. along the line of the Li and Verma's proposal in [7].

Future works includes evaluating the combination of adaptive anisotropic regularization together with multi-channel image registration, and exploring more elaborate ways to fuse the information of multiple channels

\section{Acknowledgement}

Support for this research was provided in part by the Swedish Research Council (2007-4786), AgoraLink at Linköping University and the National Institute of Health (R01MH082918, R01MH074794, R01MH092862, P41RR013218). 


\section{References}

1. Avants, B., Duda, J., Zhang, H., Gee, J.: Multivariate Normalization with Symmetric Diffeomorphisms for Multivariate Studies. In: MICCAI (2007)

2. Forsberg, D., Andersson, M., Knutsson, H.: Adaptive anisotropic regularization of deformation fields for non-rigid registration using the morphon framework. In: ICASSP (2010)

3. Guimond, A., Guttmann, C., Warfield, S., Westin, C.F.: Deformable registration of DT-MRI data based on transformation invariant tensor characteristics. In: ISBI (2002)

4. Holden, M.: A Review of Geometric Transformations for Nonrigid Body Registration. Medical Imaging, IEEE Transactions on (2008)

5. Knutsson, H., Westin, C.F.: Normalized and Differential Convolution: Methods for Interpolation and Filtering of Incomplete and Uncertain Data. In: CVPR (1993)

6. Kybic, J.: Bootstrap resampling for image registration uncertainty estimation without ground truth. Image Processing, IEEE Transactions on (2010)

7. Li, Y., Verma, R.: Multichannel Image Registration by Feature-Based Information Fusion. Medical Imaging, IEEE Transactions on (2011)

8. Park, H.J., Kubicki, M., Shenton, M.E., Guimond, A., McCarley, R.W., Maier, S.E., Kikinis, R., Jolesz, F.A., Westin, C.F.: Spatial Normalization of Diffusion Tensor MRI Using Multiple Channels. Neuroimage (2003)

9. Peyrat, J.M., Delingette, H., Sermesant, M., Pennec, X., Xu, C., Ayache, N.: Registration of $4 \mathrm{D}$ Time-Series of Cardiac Images with Multichannel Diffeomorphic Demons. In: MICCAI (2008)

10. Risholm, P., Pieper, S., Samset, E., III, W.W.: Summarizing and Visualizing Registration Uncertainty in Non-Rigid Registration. In: MICCAI (2010)

11. Rohde, G.K., Pajevic, S., Pierpaoli, C., Basser, P.J.: A Comprehensive Approach for Multi-channel Image Registration. In: WBIR (2003)

12. Studholme, C.: Incorporating DTI Data as a Constraint in Deformation Tensor Morphometry Between T1 MR Images. In: IPMI (2007)

13. Thirion, J.P.: Fast Non-Rigid Matching of 3D Medical Images. Research Report RR-2547, INRIA (1995)

14. Vercauteren, T., Pennec, X., Perchant, A., Ayache, N.: Non-parametric Diffeomorphic Image Registration with the Demons Algorithm. In: MICCAI (2007)

15. Wang, Y., Gupta, A., Liu, Z., Zhang, H., Escolar, M., Gilmore, J., Gouttard, S., Fillard, P., Maltbie, E., Gerig, G., Styner, M.: Dti registration in atlas based fiber analysis of infantile krabbe disease. Neuroimage (2011)

16. Wassermann, D., Bloy, L., Kanterakis, E., Verma, R., Deriche, R.: Unsupervised white matter fiber clustering and tract probability map generation: Applications of a Gaussian process framework for white matter fibers. NeuroImage (2010) 